\title{
PROVA DO ESFORÇO NA FORMA INDETERMINADA DA DOENÇA DE CHAGAS
}

\author{
Vanize Macedo, Raimundo Santos e Aluizio Prata
}

\begin{abstract}
Os autores realizaram a prova do esforço sub-máximo em 30 portadores da forma indeterminada da doença de Chagas e em 30 normais, a fim de avaliar a capacidade física e a função miocárdica. Os resultados nâo mostraram diferenciação do grupo positivo e do controle negativo, quanto a queixas, frequiência cardiaca ou respiratória, tensão arterial, consumo de oxigênio e alterações eletrocardiográficas.
\end{abstract}

\section{INTRODUÇÃO}

A forma indeterminada da doença de Chagas foi bem definida por Laranja (4) quando disse que ela era uma forma laboratorial. Para Carlos Chagas, nesta fase da latência que poderia durar muitos anos, os indivíduos eram "cardíacos potenciais" (1). A forma indeterminada da doença de Chagas tem grande importância médico social, pois os indivíduos nela enquadrados são às vezes incapacitados para o trabalho em testes de seleção.

$\mathrm{E}$ possivel que o número de pacientes classificados nesta forma possa diminuir à medida que os submetemos a métodos diagnósticos mais sensíveis e especializados. Neste trabalho fazemos uma tentativa para evidenciar possíveis alterações cardíacas em pacientes com a forma indeterminada da doença de Chagas.

\section{MATERIAL E MÉTODOS}

Sessenta indivíduos que vêm sendo acompanhados há anos na área endêmica para doença de Chagas, em São Felipe, foram submetidos à prova ơo esforço submáximo. Trinta com a forma indeterminada da doença de Chagas, selecionados pelo xenodiagnóstico positivo, e trinta controles negativos selecionados pelo xenodiagnóstico negativo e três reações de fixação de complemento não reagentes, em diferentes laboratórios. As idades variaram de 18 a 45 anos, sendo 15 de cada sexo nos dois grupos.

Cada pessoa tinha uma ficha protocolo com o registro de pulso, freqüência respiratória, tensão arterial, temperatura axilar, peso e altura. O paciente era colocado em repouso durante uma hora, antes do início da prova, tendo sido tomado um traçado eletrocardiográfico com as 12 derivações clássicas. Evitou-se o uso de bebidas alcoólicas e do fumo antes da prova, não sendo selecionados os indivíduos com anemia, desnutrição ou com doença manifesta. O teste de escolha foi o da "escada", (dois degraus), variante de Master, pela sua simplicidade, que permitiu o seu uso em estudo de campo. A escada pa-

\footnotetext{
* Trabalho da Fundaçăo Gonçalo Moniz (Diretor Dr. J. F. Figueiredo), da Disclplina de Doenças Infecclosas e Parasitárias da Faculdade de Medicina da Universidade Federal da Bahia e da Faculdade de Ciências da Saúde da Universidade de Brasília.
} 
TABELA 1

Manifestaçōes clínicas após prova de esforço em pacientes com forma indeterminada da doença de Chagas e em controles

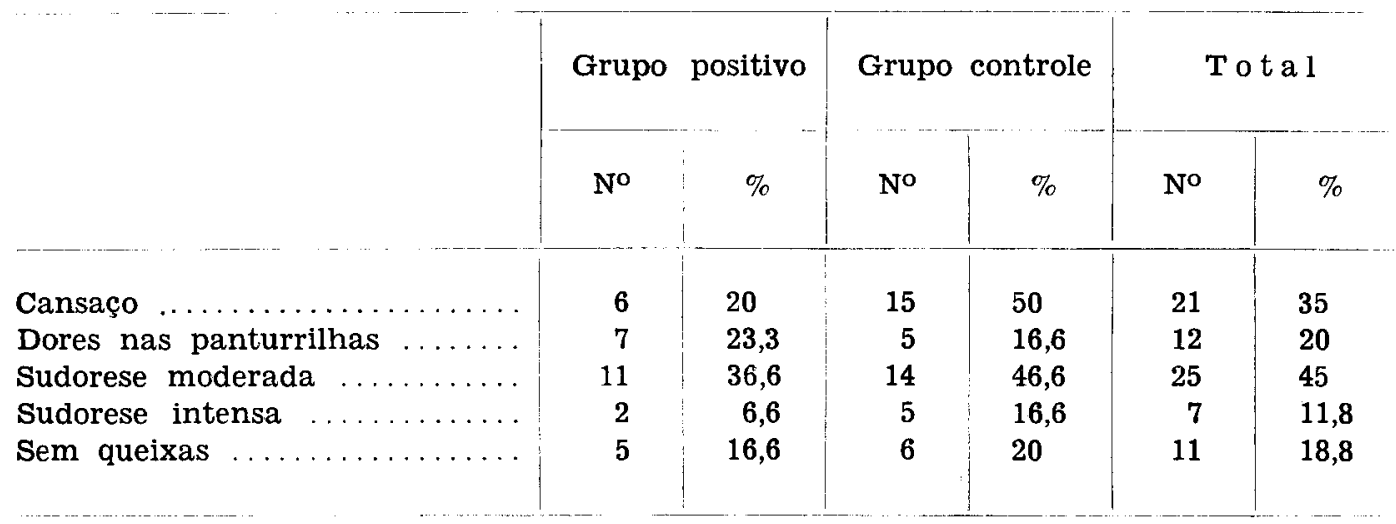

TABELA 2

Alteraçōes eletrocardiográficas após prova de esforço em pacientes com forma indeterminada da doença de Chagas e em controles

Extrassistoles atriais

Desnivelamento ST/T:

$0,5 \mathrm{~mm}$

$1 \mathrm{~mm}$

$\ldots \ldots \ldots \ldots \ldots$

Grupo positivo $\mid$ Grupo controle

Tot a 1

$1,5 \mathrm{~mm}$

1

1

1
1

2

1

3

2 
drão utilizada tinha $50 \mathrm{cms}$. de altura x 50 cms. de largura, com dois degraus de 25 cms. cada e $23 \mathrm{cms}$ de profundidade. O individuo era levado a subir e descer os dois degraus durante seis minutos, cronometrados, sendo o número de ascenções/ minuto calculado para cada um através de uma tabela de peso, idade e sexo (Shepard). O número de ascenções/minuto variou de 16 a 21.

Durante a prova foram procuradas queixas clínicas, assim como verificada a freqüência de pulso.

Imediatamente após a parada do esforço era tomado o pulso, respiração e a tensão arterial e realizado um eletrocardiograma nas derivaçōes $D_{1}, D_{2}, A V F, V_{4}$, $V_{5}$ e $V_{6}$, seguindo-se de $D_{.2}$ contínua $d u-$ rante dois minutos. Outros traçados nas derivaçōes descritas foram tomados com 6, 10 e 15 minutos. O trabalho realizado pode ser avaliado utilizando-se a seguinte fórmula $\mathrm{T}=\mathrm{P} \times \mathrm{a} \times \mathrm{s}$, Peso $(\mathrm{Kg}) \times \mathrm{a}=$ $=$ altura escada $(\mathrm{m}) \mathrm{x} \mathrm{n}^{\circ}$ de subidas no " $t$ ".

O trabalho realizado variou de 300 a $750 \mathrm{kgm} / \mathrm{m}$ que equivale mais ou menos a $1000 \mathrm{kgm} / \mathrm{m}$ na bicicleta ergométrica. 0 consumo de oxigênio foi medido de maneira indireta de acordo com a freqüência cardíaca, utilizando-se o normograma de "Astrand Ryhming" para a prova da escada. Da mesma forma o consumo máximo de oxigênio (VO.,) foi achado pela tabela de Astrand, sofrendo a correção para idade e freqüência cardíaca. Os traçados eletrocardiográficos foram interpretados a cegas, segundo os critérios da "American Heart Association”. (3, 5, 6, 7, 8, 9).

\section{RESULTADOS}

Foram realizados os seguintes parâmetros: sintomas, freqüência cardiaca, freqüência respiratória, tensão arterial, consumo máximo de oxigênio/min. e alteraçōes eletrocardiográficas (arritmias, alteraçōes da condução A-V, e intraventricular e do segmento $\mathrm{ST} / \mathrm{T}$ ) .

As principais queixas na forma indeterminada, foram: sudorese moderada em $36,6 \%$, sudorese intensa em $6,6 \%$, cansaço em $20 \%$ e dores nas panturrilhas em $23,3 \%$, comparadas respectivamente com $46,6 \%$ $16.6 \%, 50 \%$ 'e $16,6 \%$ no grupo controle ( $\mathrm{Ta}-$ bela 1). Em $16.6 \%$ dos positivos e em $20 \%$ dos negativos não houve referência a queixas.
A freqüência cardíaca depois da prova aumentou em 13 chagásicos (43\%) e em 16 não chagásicos $(56,3 \%)$ nos dois grupos, somente dois indivíduos do grupo controle alcançaram freqüência de $170 \mathrm{~b} / \mathrm{min}$. Nos dois grupos, a freqüência respiratória sofreu discreto aumento, mas logo voltou à normalidade, assim como a T.A. que se elevou em média de $20 \mathrm{~mm}$ de mercúrio, após a prova.

O consumo de oxigênio e o consumo máximo de oxigênio determinado pela tabela de Astrand Ryhming e corrigida pelo fator idade/freqüência carálaca, não mostraram diferença entre o grupo positivo e o controle (Fig. 1), não tendo ultrapassado a $5 \mathrm{lts} / \mathrm{min}$. nos dois grupos, considerados os valores médios para idade da tabela de Lange Anderson (3).

As alteraçōes eletrocardiográficas estiveram presentes em oito casos $(13,9 \%)$ sendo três (5\%) do grupo positivo e cinco $(8,3 \%)$ do grupo controle. As alterações foram extrassistoles atriais $e$ desnivelamento de ST/T de $0,5 \mathrm{~mm}$ e $1,5 \mathrm{~mm}$.

\section{DISCUSSÃO}

Com o surto de desenvolvimento industrial no Brasil, a forma indeterminada da doença de Chagas vem se tornando um problema de importância crescente em medicina de trabalho, devido ao fato de que individuos portadores desta forma de doença estão sendo freqüentemente incapacitados em testes de seleção para emprego.

As provas de esforço vêem sendo usadas com a finalidade de avaliar a função cárdicrespiratória, capacidade física dos individuos e sobretudo determinar o prognóstico de cardiopatias. Vários são os métodos empregados para esta avaliação, alguns dos quais, por serem sofisticados, só podem ser usados em laboratórios especializados.

Pela prova do esforço submáximo não conseguimos evidenciar alterações que pudessem distinguir o coração dos pacientes com a forma indeterminada da doença de Chagas dos normais usados como controle. Sem entrarmos em apreciaçōes sobre o potencial evolutivo da forma indeterminada da doença de Chagas, devemos considerar que, do ponto de vista imediato, o coração nesta forma de doeņ̧a é tão eficiente como o dos individuos normais, quando submetidos ao esforço submáximo. 
FIG. -1

\section{CONSUMO MÁXIMO DE OXIGÊNIO EM}

\section{PACIENTES CHAGÁSICOS E CONTROLES}

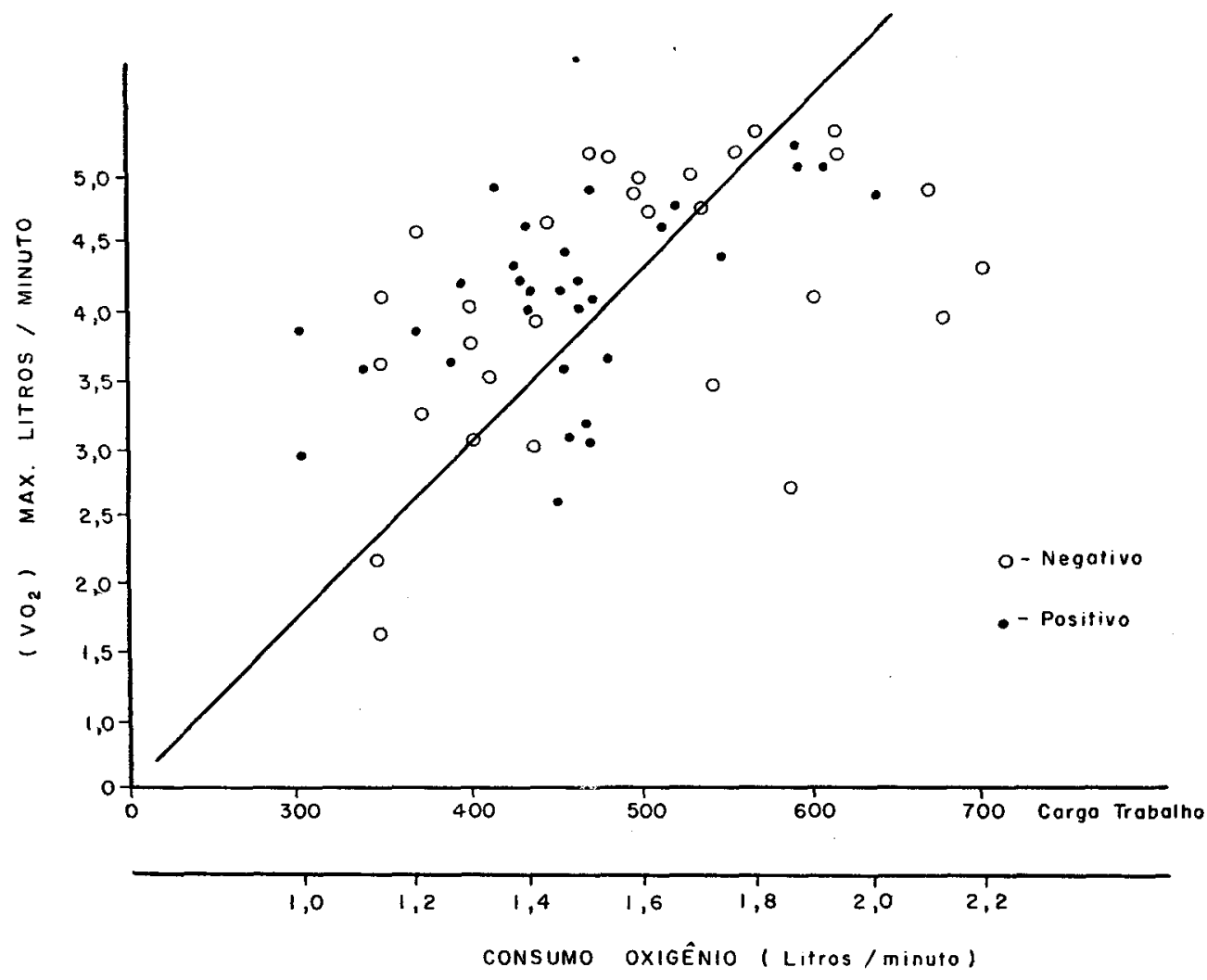




\section{SUMMARY}

To evaluate the physical capacity and the myocardial function in 30 patientes with latent Chagas' disease, the effort test was performed. 30 controls were used. According to complains, cardiac and respiratory rate, maximum oxigen consumption and eletrocardiographic changes there was no difference between both groups.

\section{REFERÊNCIAS BIBLIOGRÁFICAS}

1. CHagas, G. - Processos patogênicos da tripanossomose americana. Mem Inst. Oswaldo Cruz, 8: 5 -36, 1916.

2. DOAN, A. E.; PETERSON, D. R.; BLOCKMON, J. R \& BRUCE, R. A - Myocardial ischemia after maximal exercise in healthy men. A method for detecting potential coronary heart disease? Heart J., 69: 11-21, 1965.

3. LANGE, A. K. ; SHEPHARD, R. J.; DENOLIN, H.; VARNAUHOS, E. \& MASIRONI, R. - Fundamental of exercise testing. Who. Geneva, 1971.

4. LARANJA, F. S. - Aspectos clínicos da moléstia de Chagas. Rev. bras. Med., 10: 482-91, 1953.

5. MASTER, A. M. \& JAFFE, H. L. The electrocardiographic changes after exercise in angina pectoris. $J$. Mount Sinai Hospital, 7: 629-32, 1941.

6. _ \& ROSENFELD, L. - Clinical application of the two step exercise test. J. Amer. Med. Assoc., 178: 283, 1961 .

7. MATTINGLY, T. W. - The post exercise electrocardiogram - It's value in the diagnosis and prognosis of coronary arterial disease. Amer. J. Cardial., 9: 395, 1962 .

8. ROSE, G. \& BLACKBOURN, H. Cardiovascular survey method. Geneva, WHO Moncgraph Series no 56.

9. SHEFFIELD, L. T.; HOLF, J. H. \& REWER, T. J. - Exercise graded by heart rate in eletrocarỏiographic testing for angina pectoris. Circulation, $23,622-629,1965$. 